\title{
Investigating the shear rheology of molten instant coffee at elevates pressures using the Cambridge Multipass Rheomter
}

\author{
Michael J Sargent ${ }^{\mathrm{a}, *}$, B Hallmark ${ }^{\mathrm{a}}$ \\ ${ }^{a}$ Department of Chemical Engineering and Biotechnology, University of Cambridge, Phillipa Fawcett Drive, West Cambridge Site, \\ Cambridge
}

\begin{abstract}
The processing of instant coffee may involve pumping the material in a melt phase through operations at elevated pressures. The shear rheology of this material was investiaged using the Cambridge Multipass Rheometer, which allows for shear rheometric testing under conditions of independently controlled temperature and pressure. In this work the back pressire was set to dissolve any air that was present in the melt, so that the rheology of the single phase material could be tested. Data were collected over the accessible range of conditions; shear rates from 0.01 to $1000 \mathrm{~s}^{-1}$, temperatures from 80 to $110{ }^{\circ} \mathrm{C}$, and pressures from 0.01 to 300 bar. The melt exhibited thixotropic behaviour at low shear rates, and the data could be fitted to a non-dimensionalised Carreau fluid model with a temperature dependence which followed an Andrade relationship. Sample to sample variation was observed, which is attributed to differing water content. The results demonstrate how the rheology of complex food materials can be accessed under process conditions.
\end{abstract}

Keywords: Rheology, Coffee, Process conditions, Thixotropy, Carreau model

\section{Introduction}

Coffee is consumed worldwide in many different forms, with regional preferences on the forms of coffee sold and prepared. The most obvious distinction is between freshly brewed coffee and 'instant' coffee, a general term used to mean any form of coffee that requires no special equipment to create the beverage. Worldwide, the instant coffee market is valued at over $\$ 30$ billion, and contributes over $40 \%$ of the total coffee consumed $[27,29]$. This is more than half a million tons of instant coffee produced every year.

Instant coffee itself comes in many forms and processes used, the primary two being granules from freeze drying, or powder from spray drying. There are also liquid forms of instant coffee that fall under the definition, however we shall only concern ourselves with the solid forms for the purposes of this paper. The drying processes typically reduce the water content of commercial products to $<5 \%$ by mass, the remainder of which is a mixture of carbohydrates, proteins, flavour oils, phosphates, and melanoidins $[23]$.

After the drying process, the solid material may have to be further processed to create the commercial product. These are usually thermal and/or mechanical processes which leave the composition unchanged, to create a product with more customer appeal. Examples of such processes are agglomeration, granulation, and melt extrusion. There is little academic literature on these particular

\footnotetext{
* Corresponding author

Email addresses: mjs244@cam.ac.uk (Michael J Sargent), bh206@cam.ac.uk (B Hallmark)
}

applications, likely due to a desire for secrecy in the instant coffee industry. There are however a number of patents for processing instant coffee as a molten material [1, 20, 31].

The purpose of this research is to perform an investigation into the rheological characteristics of instant coffee melts. By identifying the key rheological properties which will affect processing, an understanding can be reached which does not require the exact viscous response to be known under all possible conditions. This will allow processing involving the melt phase processing of instant coffee to be better understood, and to allow for the modelling and optimisation of such unit operations.

\section{Materials and Methods}

There are many established techniques and protocols that can be used to characterise the rheology of complex fluids. Examples include rotational rheometry [17], making use of parallel plates, cone and plate or Couette geometries, capillary rheometry [25], and vibrational rheometry [9]. The aim of the work presented in this paper is to investigate the rheology of single phase molten instant coffee; this presents a significant challenge since air inclusions are unavoidable in the preparation of the melt phase since the melt derives from a powdered granular solid.

One way in which the single phase rheology can be investigated, however, is to carry out characterisation work at an elevated pressure such that the included air is allowed to dissolve into the melt phase. Experiments of this nature require the use of a rheometer that has complete sample containment, such that the sample can be held at 
pressures typical of process equipment. One rheometer in particular provides this capability the Cambridge multipass rheometer (MPR) [14].

\subsection{The multipass rheometer}

The Cambridge multipass rheometer (MPR) is a fullyenclosed, twin-piston, capillary rheometer capable of operating at elevated temperature and pressure. It has been specifically designed to be modular and operate with a wide variety of capillary test sections, which include geometries that can be interrogated optically [8] and by $\mathrm{x}$ ray diffraction [12]. This makes the MPR an ideal platform with which to investigate the rheological response of a broad range of materials. A schematic diagram of a typical fluid pathway within the MPR is shown in Figure 1.

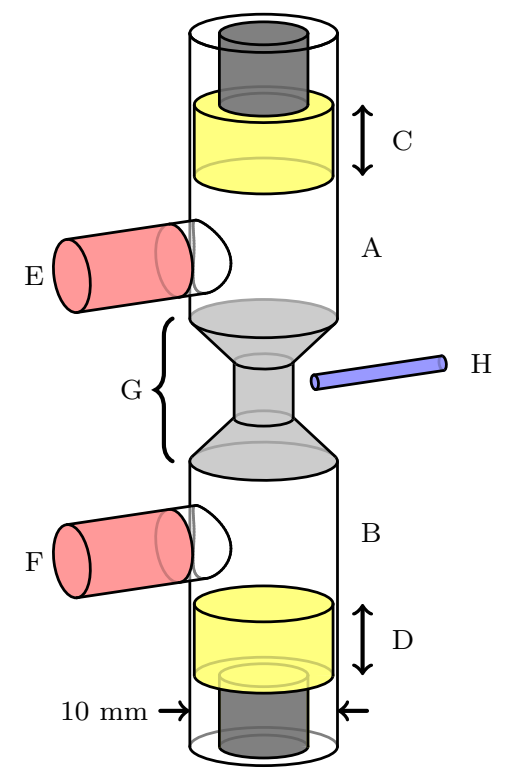

Figure 1: Multipass rheometer internal space

$A \& B$ - fluid reservoirs, $C \& D$ - driving pistons,

$E \& F$ - pressure transducers and thermocouples, $G$ - capillary test section, $H$ - auxiliary thermocouple

(intervening space is stainless steel).

The fluid under test occupies the fluid reservoirs (A and B) and the space within the capillary test section (G). In use, two independently-controlled servo-hydraulic pistons ( $\mathrm{C}$ and D) move the fluid through the capillary test section, and the pressure drop across the test section is recorded by two pressure transducers ( $\mathrm{E}$ and $\mathrm{F}$ ). An optional thermocouple $(\mathrm{H})$ can be placed to record the temperature of the fluid in the capillary test section. Due to the independent nature of each piston, the fluid within the reservoirs and capillary test section can either be pressurised by moving the pistons towards each other, or depressurised by moving the pistons away from each other.

A further advantage of the fully enclosed nature of the MPR is the ability to carry out multiple tests on the same sample of fluid: this allows investigation of the effect that strain history has on the rheological properties of a material, revealing phenomena such as thixotropy. The adaptable nature of the MPR has allowed study of single phase materials such as polymer melts $[15,19,11]$, multiphase materials such as polystyrene foams [28] and foamed starch [18], and more complex multiphase materials such as model ice cream systems [16].

Each of the fluid reservoirs shown in Figure 1 is controlled by a hydraulic piston, which can be operated independently. This allows for a wide range of possible tests to be performed by the same piece of equipment. The assembly is modular, such that the transducers $E$ and $F$, and the capillary test section $G$ can be selected for the most appropriate geometry for the fluid to be tested. When testing molten instant coffee, the transducers used could measure 0.01 to 200 bar, and the capillary section was $4 \mathrm{~mm}$ in diameter and $4 \mathrm{~mm}$ long. Such a short, wide capillary was necessary due to the extremely high viscosity of the sample.

Two main types of test were performed on the molten coffee: the first was a compressibility test, where both pistons were moved towards each other in a synchronous manner. This increased the pressure of the sample without causing any net flow and allowed the pressure volume relationship of the coffee melt to be investigated, revealing the point where the fluid transitioned from a two phase bubbly liquid to a single phase melt.

The second test that was carried out is termed a 'combination control shear test'. Here, the piston in the fluid reservoir being used as the fluid source extends at a specified velocity, causing a known volumetric flow of material through the capillary test section. The piston in the fluid reservoir into which the capillary test section discharges moves under pressure control, maintaining a specified pressure in the downstream reservoir. The aim of the 'combination control shear test' is to provide known flow conditions such that pressure drop can be measured and viscosity calculated as a function of applied shear rate, but in such a manner that a dissolved gas can be kept in solution under pressure. For materials such as molten instant coffee, this ability is a vital pre-requisite for any testing protocol.

\subsection{Sample preparation protocol and testing conditions}

In order to be able to test a number of different coffee samples such that comparisons between sample behaviour can be made, it is necessary to develop a standardised sample preparation protocol. The aim of the sample preparation protocol is to ensure that the included air is kept to a pragmatic minimum, that the mechanical integrity of the MPR pressure transducers is not inadvertently compromised, and that thermal decomposition of the coffee melt is avoided. A schematic diagram of the sample preparation protocol is shown in Figure 2.

Firstly, the fluid reservoirs and capillary test section of the MPR were filled with finely ground coffee powder. 


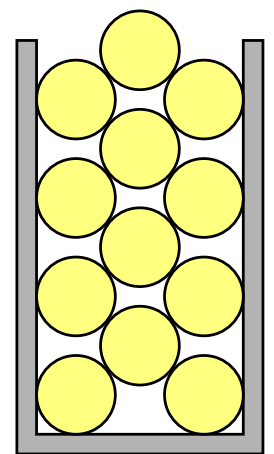

(a)

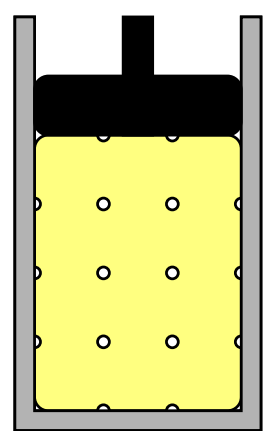

(c)

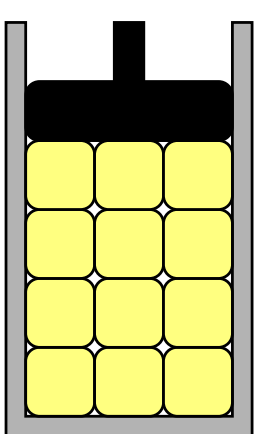

(b)

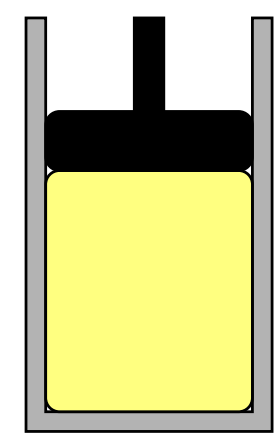

(d)
Figure 2: Loading Protocol

(a) - test apparatus is loaded with granules or powder. (b) - material is compacted to reduce voidage. (c) - test apparatus is heated to form a bubbly melt. (d) - melt is further compressed to dissolve gas.

The powder was then compacted, manually at first using a brass rod and subsequently by the hydraulic pistons. The compaction step acts to reduce the voidage of the material and hence the volume of air trapped in the melt.

The compacted powder was then heated to $110{ }^{\circ} \mathrm{C}$ such that the particles start to melt. This step required great care as it is possible to suddenly overload the pressure transducers and cause substantial mechanical damage. This is due to the compacted powder being able to form a stable bridge in the tappings for each of the pressure transducers, thus preventing the pressure transducers from reading the true pressure that the pistons apply to the powder. When the powder starts to melt, any powder bridges that may be present yield and the pressure transducers are exposed to the bulk melt pressure, which can cause damage. To prevent this, the pre-melt compaction is limited to 10 bar. Lastly, the bubbly melt is further compressed to dissolve the air bubbles in the molten instant coffee. This gives a single phase melt suitable for rheological testing.

The temperature range for testing was chosen to be representative of the likely temperatures under which molten instant coffee may be processed. A range of $70-110^{\circ} \mathrm{C}$ was selected, based on the intended operating ranges of a num- ber of patented processes $[2,3]$. While rationale for these upper and lower bounds is not given in the patents, it is reasonable to assume that they are due to thermal degradation of the food material and mechanical limitations of the processing equipment respectively. In the MPR samples were first tested at $110^{\circ} \mathrm{C}$ at which the viscosity was lowest, and the temperature was lowered for subsequent tests until the viscosity became too high for the apparatus.

For the rheological investigation presented in this paper, the MPR was equipped with pressure transducers that could measure up to 200 bar, and a precision of 0.01 bar bar with sufficient data analysis. Compressibility tests were carried out using a capillary test section the same diameter as the MPR pistons, $10 \mathrm{~mm}$, to minimise viscous contributions to the recorded pressures. Shear rheology testing used a capillary test section $4 \mathrm{~mm}$ in diameter and $4 \mathrm{~mm}$ long; these dimensions were chosen due to the very high viscosity of the melt phase. The downstream reservoir for shear tests was held at different back pressures for different tests, ranging from 20 - 60 bar.

The pistons which drive the flow could be operated at velocities ranging from $0.001-100 \mathrm{mms}^{-1}$. For each sample at each temperature, tests were carried out in increasing piston velocity, and hence increasing strain rate, starting at $0.001 \mathrm{~mm} \mathrm{~s}^{-1}$ and increasing logarithmically, with the same number of tested velocities every decade. The maximum velocity depended on the viscosity of the sample, such that the pressure recorded in the upstream reservoir did not exceed the operating range of the transducer.

The first test that was carried out was a compressibility test, such that the minimum pressure to dissolve the intergranular air could be determined. The sample was held at $110{ }^{\circ} \mathrm{C}$ and the pistons both extended at $0.1 \mathrm{~mm} \mathrm{~s}^{-1}$, until near the pressure limit for the transducers. The pistons were returned to their starting positions, and sample was then reduced in temperature by $5{ }^{\circ} \mathrm{C}$ before repeating the process. This test set a lower bound on the back pressure that was used for the subsequent sequence of shear tests, which were carried out using the combination control shear test mode on the MPR.

The sequence of shear tests for a sample started at the first specified temperature, and measured the pressure drop across the capillary test section at a series of increasing piston velocities starting with $0.001 \mathrm{~mm} \mathrm{~s}^{-1}$. When the maximum allowable pressure or pistol velocity had been attained, the MPR pistons were stopped, the sample temperature reduced to the next specified temparature, and the pressure drop measurements repeated as a function of increasing piston speed, restarting from $0.001 \mathrm{mms}^{-1}$. The lower bound on the sample temperature was deemed to have been reached when the pressure drop across the capillary test section at low piston velocity became close to the maximum working pressure of the transducers. Samples were tested in order of increasing strain rate to minimise the effect of strain history on the material. A rest 
period of at least 30 minutes was allowed for the sample to relax between the end of the shear rate sweep at one temperature and the start of the next shear rate sweep at a lower temperature.

\section{Theory}

The theory underpinning capillary rheometry is well established (see, for example, Ballman and Brown [5], Cheremis noff [7]), hence this section serves to highlight the key results that will be used to analyse data from the MPR. For a more detailed treatment of the fundamentals of capillary rheometry in the MPR, the reader is advised to consult Mackley[13].

When a fluid flows at a steady volumetric flow rate through a long narrow capillary it experiences a shear strain rate, $\dot{\gamma}$. It is usual to make reference to the shear strain rate of the fluid at a known location, typically the wall of the capillary. This wall shear strain rate, $\dot{\gamma}_{w}$, can be related to the volumetric flow rate of the fluid, $Q$, the radius of the capillary, $R_{c}$, and the Rabinowitsch correction factor, $n^{\prime}$, which accounts for the unknown strain rate dependency of the shear stress [24]. In the case of the MPR, the volumetric flow rate of the fluid can be related to the velocity of the piston driving the flow, $v_{p}$, and the radius of the piston $R_{p}$ :

$$
\dot{\gamma}_{w}=\frac{v_{p} R_{p}^{2}}{R_{c}^{3}}\left(3+\frac{1}{n^{\prime}}\right)
$$

The Rabinowitsch correction factor is a material property that can be calculated by comparing the pressure drop, $\Delta P$, across the capillary at different volumetric flow rates, hence different piston velocities, $v_{p}$ :

$$
n^{\prime}=\frac{d \log (\Delta P)}{d \log \left(v_{p}\right)}
$$

The pressure drop across the capillary test section can also be related to the shear stress in the fluid by means of a force balance. Again, it is usual to make reference to the shear stress at a known location, typically the wall of the capillary. The resulting force balance allows the wall shear stress, $\tau_{w}$, to be related to the pressure drop across the capillary test section, $\Delta P$, the capillary radius, $R_{c}$, the capillary length, $L_{c}$, and the Bagley correction factor, $\alpha$, which accounts for entry and exit losses in the capillary. It is usual to express $\alpha$ as a number of extra capillary radii that need to be added to the capillary length to compensate for these losses [4]:

$$
\tau_{w}=\frac{\Delta P R_{c}}{2\left(L_{c}+\alpha R_{c}\right)}
$$

$\alpha$ is more complicated to calculate than $n^{\prime}$, as it depends on both material properties and flow geometry; for this work $\alpha$ was determined via an iterative analyticalcomputational approach. An initial guess of the sample rheology was obtained by setting $\alpha=0$. Ansys Polyflow was then used to model the fluid flow and hence calculate the pressure losses for the pressure transducer locations and the assumed rheology. This allowed the value of $\alpha$ to be corrected with respect to it's initial value, and a second iteration carried out. For all samples tested, it was found that a single iteration was sufficient to ensure convergence within the error bars of the experimental measurements, -and that sample-to-sample variation of $\alpha$ was lower than these errors. Therefore the final data analysis was always calculated using a value of $\alpha=2.08 \pm 0.49$. Details of the Polyflow model are given in Appendix Appendix A. Wall shear stress is converted into apparent viscosity, the ratio of shear stress to shear strain rate, for subseqent anaylsis:

$$
\eta=\frac{\tau_{w}}{\dot{\gamma}_{w}}
$$

The analysis that has just been outlined assumes that the flow of the fluid through the capillary is steady: in the MPR there is, however, a finite time over which flow can happen before it must reverse, due to the maximum extent that the pistons can move. To reduce experimental error, each pressure drop data point given in the results section is the average of 5 passes of the pistons. The piston velocity determines the length of time for each pass, as each piston has a maximum of $30 \mathrm{~mm}$ travel. All piston velocities below $3 \mathrm{~mm} \mathrm{~s}^{-1}$ received $10 \mathrm{~s}$ of flow for each pass, but at higher piston velocities the duration of each pass decreased. Between each of the passes, the fluid was left to rest for $10 \mathrm{~s}$.

\section{Results and discussion}

In total, 8 samples of molten instant coffee were tested using the MPR. The experimental conditions for all 8 samples are summarised in Table 1.

\subsection{Compressibility}

A plot of sample volume as a function of applied pressure at temperatures ranging between $65{ }^{\circ} \mathrm{C}$ and $110{ }^{\circ} \mathrm{C}$ is shown in Figure 3. In this plot, the volume of the sample was calculated from the volume of the capillary and the positions of each piston. The combination of slow piston velocity and large capillary radius ensured that the pressure that was measured was due to sample compression alone, rather than a combination of pressure due to compression and the effect of any shear flow.

Two regimes of behaviour are evident in Figure 3. At pressures below 25 bar, a small change in pressure has a relatively large effect on sample volume, whereas above 25 bar the pressure dependence of sample volume is less pronounced, and samples have an average compressibility value of $2.88 \times 10^{-9} \mathrm{~Pa}^{-1}$.

The inset photograph in Figure 3 is an optical transmission micrograph that shows the microstructure of a sample of molten coffee at a pressure of 1 bar absolute. The dark 
Table 1: Successful shear tests of molten instant coffee

\begin{tabular}{|c|c|c|c|c|c|c|}
\hline sample number & test objective & series & temperature $\left[{ }^{\circ} \mathrm{C}\right]$ & back pressure [bar] & velocities tested & notes \\
\hline 0 & compressibility & - & - & - & - & not a shear test \\
\hline \multirow{6}{*}{1} & \multirow{6}{*}{ back pressure } & $\mathrm{a}$ & 110 & 20 & 6 & not pre sheared \\
\hline & & $\mathrm{b}$ & 110 & 40 & 6 & \\
\hline & & $\mathrm{c}$ & 110 & 60 & 6 & \\
\hline & & d & 100 & 20 & 7 & \\
\hline & & e & 100 & 40 & 7 & \\
\hline & & $\mathrm{f}$ & 100 & 60 & 6 & \\
\hline \multirow{4}{*}{2} & \multirow{4}{*}{ temperature } & $\mathrm{a}$ & 110 & 40 & 14 & not pre sheared \\
\hline & & $\mathrm{b}$ & 100 & 40 & 12 & \\
\hline & & $\mathrm{c}$ & 90 & 40 & 10 & \\
\hline & & $\mathrm{d}$ & 80 & 40 & 5 & \\
\hline \multirow{5}{*}{3} & \multirow{5}{*}{ fine temperature } & $\mathrm{a}$ & 100 & 40 & 9 & not pre sheared \\
\hline & & $\mathrm{b}$ & 95 & 40 & 10 & \\
\hline & & $\mathrm{c}$ & 90 & 40 & 9 & \\
\hline & & d & 85 & 40 & 8 & \\
\hline & & $\mathrm{e}$ & 80 & 40 & 5 & \\
\hline \multirow{2}{*}{4} & \multirow{2}{*}{ hysteresis } & $\mathrm{a}$ & 110 & 40 & 25 & increasing shear rate \\
\hline & & $\mathrm{b}$ & 110 & 40 & 24 & decreasing shear rate \\
\hline \multirow{3}{*}{5} & \multirow{3}{*}{ repeat testing } & $\mathrm{a}$ & 110 & 40 & 25 & not pre sheared \\
\hline & & $\mathrm{b}$ & 110 & 40 & 25 & \\
\hline & & $\mathrm{c}$ & 110 & 40 & 25 & \\
\hline 6 & repeat testing & $\mathrm{a}$ & 100 & 40 & 25 & could not continue \\
\hline 7 & repeat testing & $\mathrm{a}$ & 110 & 40 & 20 & could not continue \\
\hline
\end{tabular}

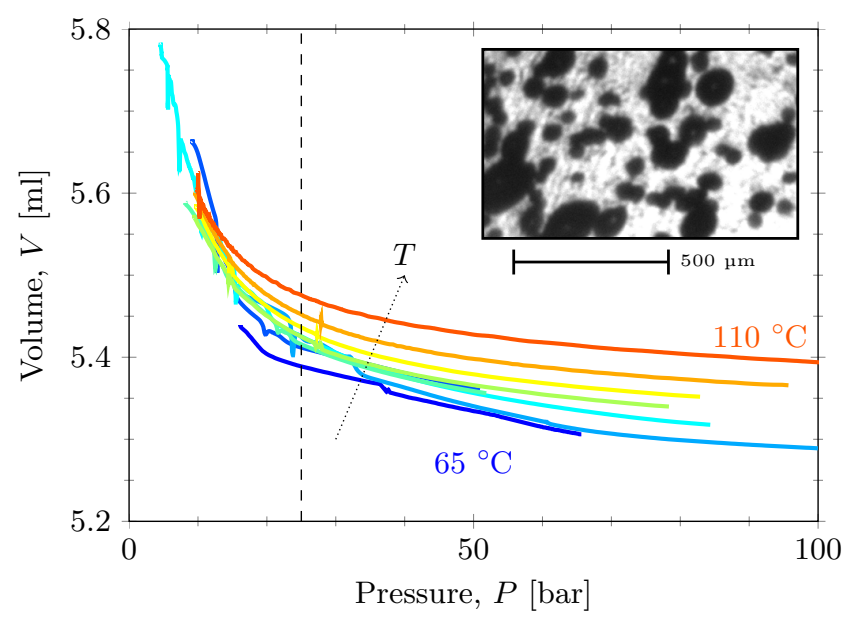

Figure 3: Compressibility

Pressure-volume relationship for a sample of molten instant coffee at different temperatures from $65^{\circ} \mathrm{C}$ to $110^{\circ} \mathrm{C}$ in steps of $5{ }^{\circ} \mathrm{C}$. Inset - transmission microscopy of $500 \mu \mathrm{m}$ thick sample, dark regions are refraction from bubbles.

regions in this image correspond to bubbles of air; the dark colouration results from light refracting around the bubbles. This photograph demonstrates that gas bubbles are present in the melt, as anticipated, at low pressure and explains why the compressibility of the coffee samples is significantly greater at lower pressures than at higher pressures.

Compressibility testing demonstrated that a minimum pressure of 25 bar is required to ensure that all the air bubbles within the coffee melt have dissolved. This sets a lower bound for the pressure specification for the shear rheology tests.

\subsection{Shear rheology}

Two key observations were made from the data obtained for the shear rheology tests. The first observation was that the first series of experiments on a particular sample at increasing shear strain rate gave subtly different data when compared to subsequent shear tests using the same conditions on the same sample. The second, and most general, observation was that all the samples of molten instant coffee were shear thinning. These two observations will now be presented and discussed in detail.

The difference in shear behaviour that was observed between the first series of experiments on each sample and the subsequent series' suggests the presence of thixotropy. This phenomena was investigated prior to the analysis of the entire sample set, and is shown in Figure 4.

The data presented in Figure 4 shows how the viscosity of one sample, sample 5 from Table 1, changes as a function of shear strain rate across three different increasing shear strain rate tests, all performed at the same temperature and back pressure. Data from the first increasing shear strain rate sweep, experiment 5a, shows markedly different low shear strain rate behaviour when compared to the repeated experiments, $5 \mathrm{~b}$ and $5 \mathrm{c}$.

The subset of data labelled $\mathrm{A}$ in Figure 4 is a feature present only in the first shear strain rate sweep; it is a rapid increase in viscosity as the shear strain rate approaches zero. This is similar to the behaviour expected in the presence of a yield stress, which would exhibit an inverse relationship between shear rate and apparent viscosity. However the gradient of this subset of data is approximately -0.5 , instead of the expected gradient of -1 


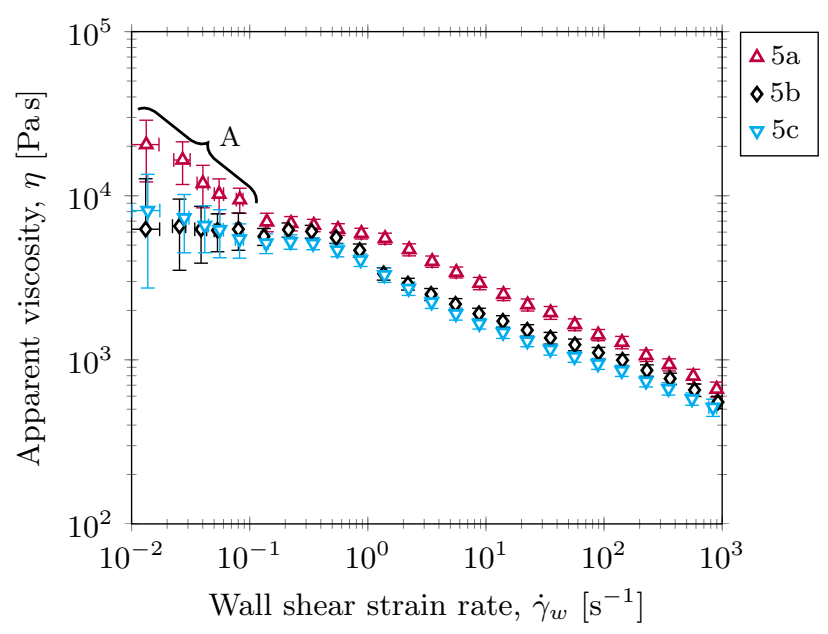

Figure 4: Thixotropy

Repeat testing of a single sample of molten instant coffee at the same temperature. Each of the three series was performed in increasing strain rate order.

for yielding behaviour. Molten crumb chocolate is another food material that has been shown to have noticeable yield behaviour at low strain rates [26], with similar shear thinning behaviour to this melt. However, in molten crumb chocolate this yield behaviour is a result of particle interactions in a dense suspension.

Marmite ${ }^{\mathrm{TM}}$ is a yeast extract spread which has similar thixotropic behaviour to this very low shear rate region, where a sample that initially appears to be shear thinning shows a more constant viscosity upon repeat testing [30]. In Marmite ${ }^{\mathrm{TM}}$ this is theorised to be due to the presence of a structure that is affected by shear; molten instant coffee may also have temporary internal structure which is destroyed by the initial shear of the sample and does not reform on the time scale of the testing procedure.

The pre-sheared rheology of molten instant coffee is more important to the melt processing of the material, as the region of any process equipment in which the unsheared rheology is applicable is negligible. As such, further analysis of the shear rheology of molten instant coffee will be restricted only to those data series after the initial series, and series 'a' in Table 1 for each sample will be ignored as unrepresentative of the pre-sheared material. This results in only 4 usable samples, as samples 6 and 7 in Table 1 were only subject to a single series each due to equipment malfunctions following their initial testing series. Additionally, sample 4 in Table 1 was tested in decreasing shear strain rate on its subsequent series of tests, and will be overlooked for this analysis as all other series were performed in increasing shear strain rate. Figure 5 shows all the rheological data from these series.

Each of the series shown in Figure 5 exhibit the same trend for shear thinning with a low shear plateau, although the magnitudes vary with both sample and temperature. A suitable constitutive equation for such behaviour

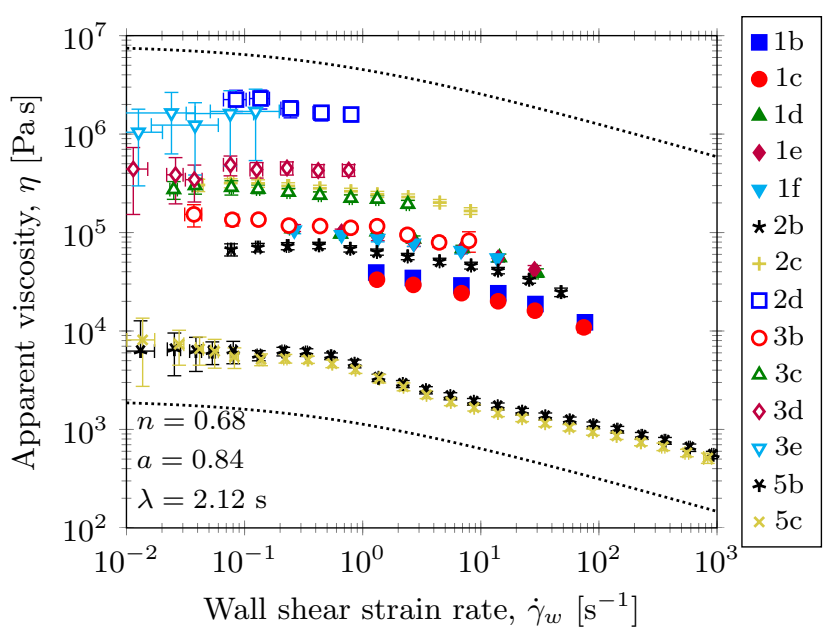

Figure 5: Shear rate dependence of viscosity Four different samples each at multiple series of increasing strain rate. Only data from pre-sheared samples is shown, to avoid thixotropic effects. The Carreau-Yasuda fit parameters shown are constant, with different $\eta_{0}$ for each series. The dotted lines show the general dependence on strain rate

is the Carreau-Yasuda model, given by equation 5. The MPR is incapable of testing this material at high enough shear rates to determine if there is a high shear Newtonain plateau, so it is assumed $\eta_{\infty}=0$. This leaves a four parameter model, where $\eta_{0}$ is the low shear Newtonian viscosity (zero shear viscosity), $\lambda$ is the transition time from Newtonian to shear thinning, $a$ is the transition sharpness, and $n$ is the shear thinning exponent.

$$
\eta(\dot{\gamma})=\left(\eta_{0}-\eta_{\infty}\right)\left(1+(\lambda \dot{\gamma})^{a}\right)^{\frac{n-1}{a}}+\eta_{\infty}
$$

For all of the samples shown in Figure 5, a single data fit is used to get single values for $\lambda, a$, and $n$, but allowing $\eta_{0}$ to vary for each series and sample. The goodness of this fit can be seen in Figure 6, where each of the series from Figure 5 is non dimensionalised against its own fitted $\eta_{0}$, and plotted alongside the trend line, and has a correlation coefficient of $R^{2}=0.922$. This means that molten instant coffee exhibits self similar behaviour across different samples and different temperatures.

\subsection{Temperature Dependence}

It has been seen that there is self-similar behaviour over different temperatures and samples. Between samples, the variation is likely to be the result of differing composition, in particular the moisture content will have a strong plasticising effect on the melt $[21,22]$. Within a single sample, there is a temperature variation of the viscosity, which is to be investigated. Only two of the samples were tested a sufficient number of times to have enough pre-sheared data series for trend line fitting, samples 2 and 3 from Table 1.

Figure 7 shows the zero shear viscosities for each of the 14 data series shown in Figure 6 against temperature. 


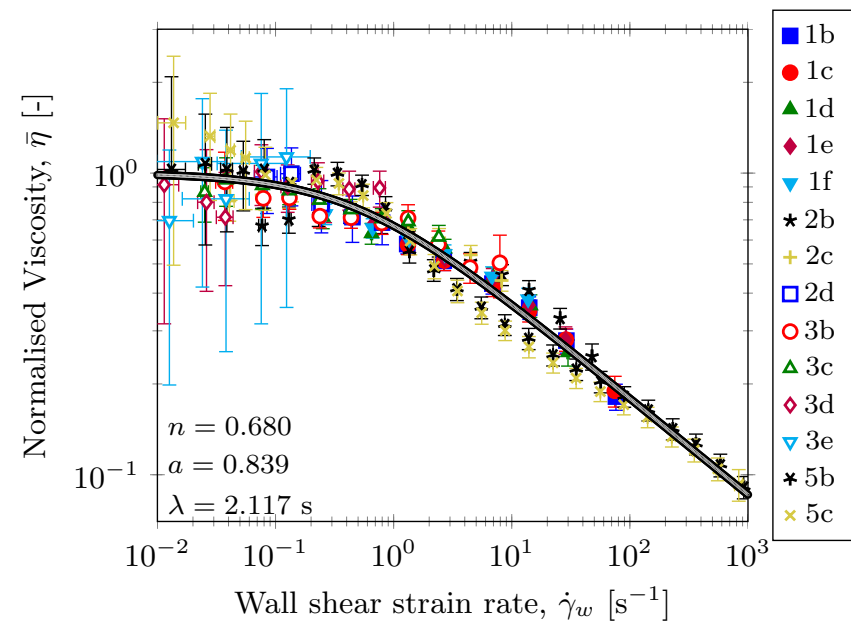

Figure 6: Nondimensionalisation of viscosity

Data from Figure 5, divided by the fitted zero shear viscosity, $\eta_{0}$, for each series.

The relationships observed are not linear, but follow the behaviour seen by Fogel'son and Likhachev [10] in polymeric glasses. This relationship between temperature and viscosity is given by Equation 6, and is similar to the Andrade equation, with the inclusion of a temperature offset.

$$
\eta_{0}(T)=A_{\eta} \exp \left(\frac{T_{\eta}}{\left(T-T_{s}\right)}\right)
$$

This is a three parameter model, with a prefactor, $A_{\eta}$, an exponent temperature, $T_{\eta}$, and a reference (solidification) temperature, $T_{s}$. The solidification temperature is that at which the viscosity tends to infinity, which Fogel'son and Likhachev [10] observed is often approximately the materials glass transition temperature for polymer melts.

Table 2: Fitted temperature dependent parameters

\begin{tabular}{cccc}
\hline sample number & $A_{\eta}[\mathrm{Pas}]$ & $T_{\eta}\left[{ }^{\circ} \mathrm{C}\right]$ & $T_{s}\left[{ }^{\circ} \mathrm{C}\right]$ \\
\hline \hline 2 & 21.57 & 552.7 & 34.9 \\
3 & 1083 & 238.0 & 46.7 \\
\hline
\end{tabular}

Table 2 shows the fitted parameters. The glass transition temperature for molten instant coffee was checked using a Perkin Elmer Pyris 1 differential scanning calorimeter (DSC) with several samples, and found to generally fall in the range $60-80{ }^{\circ} \mathrm{C}$. These were not the same samples as used in the MPR, due to the eventual destructive nature of MPR testing. This is higher than the solidification temperatures fitted, but not wildly different. $A_{\eta}$ is the predicted viscosity at infinite temperature, although extrapolation of the model beyond the tested temperature range should be avoided because the material will undergo thermal degradation. For the same reason, although the model lines crossover at around $104{ }^{\circ} \mathrm{C}$, the individual samples may not.

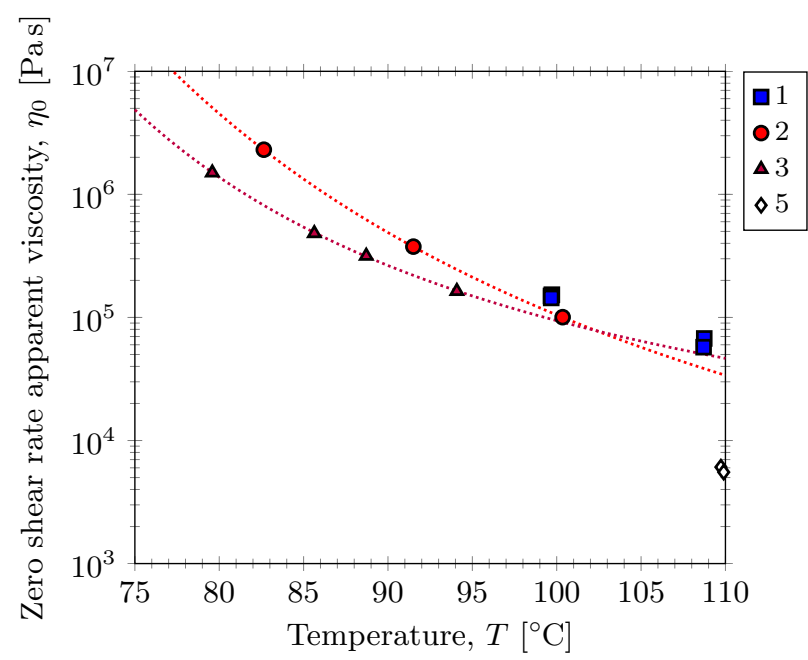

Figure 7: Temperature variation

Zero shear viscosities for two different samples. The dashed lines are Andrade type fits for the data.

\subsection{Self similarity}

It has been shown that the rheology of molten instant coffee is sensitive to temperature and sample variation, but obeys a self similar dependency on shear strain rate. Therefore for an arbitrary sample at any temperature, a single test gives its apparent viscosity and shear strain rate, which can be converted into the zero shear viscosity for that sample by rearranging Equation 5, since all other parameters are constant between samples. The difficulty is that for a single test, the Rabinowitsch correction factor $n^{\prime}$ cannot be calculated by the method given in Section 3. However for a Carreau-Yasuda fluid it can be deduced that $n^{\prime}$ must always take a value between 1 , which would be the case for purely Newtonian fluid, and the value of the shear thinning parameter $n$, which would be the expected behaviour for a purely shear thinning fluid obeying a power-law viscosity relationship. In practice, the difference between these two cases is small, as can be seen in Figure 8.

Figure 8 shows this procedure applied to the pre-sheared series of sample 2. Each MPR test gives two predicted values of $\dot{\gamma}_{w}$, depending on the chosen limit for $n^{\prime}$, and each gives a separate prediction for $\eta_{0}$. The higher prediction is always for the Newtonian case of $n^{\prime}=1$, which is generally the better prediction for low shear rate cases. The opposite is true of the shear thinning case when $n^{\prime}=0.68$. Overall, this method gives a similar order of magnitude, but a much more reliable estimate of the zero shear viscosity is obtained when analysing an entire series of data, as would be expected.

\section{Conclusions}

Molten instant coffee is a very intractable material, both in the difficulty in creating viable samples for testing, 


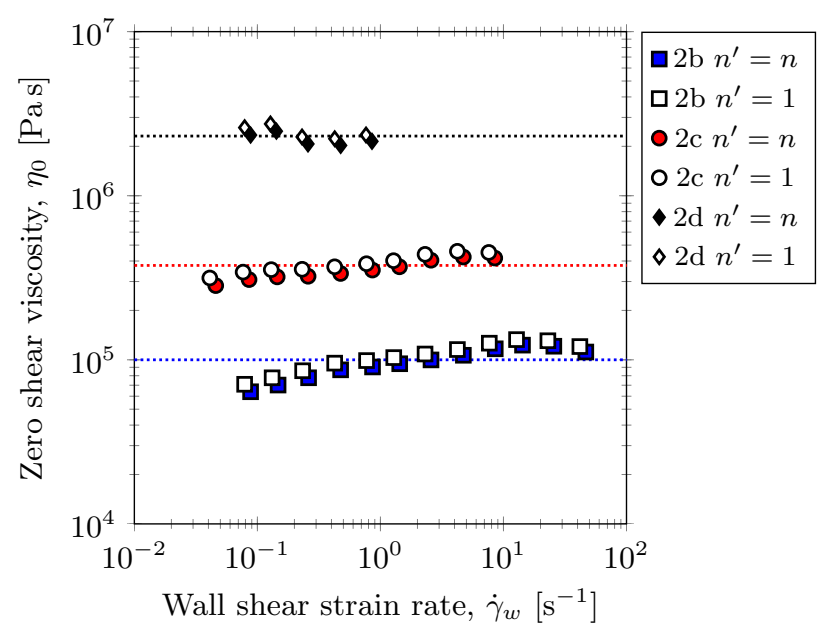

Figure 8: Self similar single test predictions

Predictions of zero shear viscsoity from single test data. Error bars are smaller than symbols

and in the forces required due to its high viscosity. Most rheometers would not be able to cope with the material, and the Cambridge Multipass Rheometer is therefore necessary. The techniques developed in this paper may be applied to the rheological testing of similar food materials with similar processing constraints.

It has been observed that the melt is thixotropic, that exposing a sample to high shear strain rates alters the viscosity at low shear strain rates. The recovery from this change has not been investigated, due to the destructive nature of testing preventing repeat tests. The viscosity of unsheared samples indicates the presence of a temporary internal structure which is destroyed by the initial shearing of the sample. Subsequent testing shows that molten instant coffee may be modelled using the Carreau-Yasuda fluid model, with some common parameters across different samples and temperatures, showing self similarity.

The variation in the viscosity between temperatures can be explained easily using the Andrade type temperature dependence proposed by Fogel'son and Likhachev [10], which includes solidification by predicting infinite viscosity. The fitted solidification temperatures are below the experimentally determined glass transition temperatures, although MPR tests were not able to be carried out over the glass transition region due to the limits of the pressure transducers.

There is also significant variation in viscosity between samples, likely due to plasticisation by water content. The legal requirement for commercial instant coffee is $<5 \%$ water by mass, although in practice it may be as low as $2 \%$, due to the difficulty in controlling the drying processes. Therefore over this range of conditions the water content is expected to cause the large change in viscosity that was observed.

\section{Appendix A. Bagley correction}

To obtain a value for the Bagley correction factor for the shear rheology of molten instant coffee, computational fluid dynamics (CFD) simulations were performed. These simulations required knowledge of the fluid rheology, which required analysis of the rheology data, which in turn required a value for the Bagley correction factor. Hence this method is iterative, repeating until a stable value of $\alpha$ is reached. Figure A.9 shows the geometry and mesh which was used in the ANSYS Polyflow simulations.

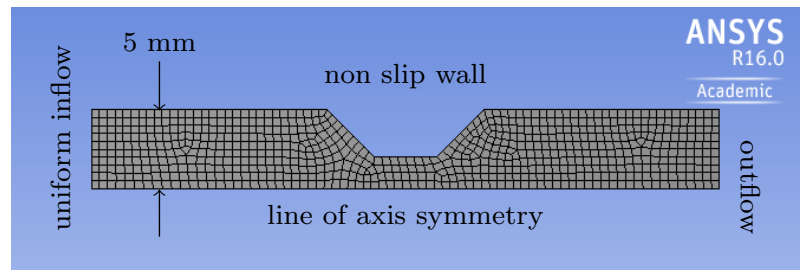

Figure A.9: Mesh for ANSYS Polyflow

2D axis symmetric MPR capillary with 17576 nodes, every polygon shown is subdivided into 25 smaller polygons for the simulation.

The geometry is two dimensional, with cylindrical axissymmetry about the centerline. The dimensions are those of the inside of the MPR using the capillary that was used in the rheological tests; capillary radius $R_{c}=2 \mathrm{~mm}$, capillary length $L_{c}=4 \mathrm{~mm}, 45^{\circ}$ entry and exit, piston radius $R_{p}=5 \mathrm{~mm}$, and transducer ports $14.7 \mathrm{~mm}$ from the ends of the angled sections. The inflow is a uniform velocity profile of $v_{p}=0.01 \rightarrow 10 \mathrm{mms}^{-1}$. The fluid is modelled as obeying perfect power law behaviour as given by equation A.1, with consistency index $k=1000 \mathrm{~Pa} \mathrm{~s}^{\mathrm{n}}$ and variable powerlaw exponent $n=0.2 \rightarrow 1$, and a density $\rho=1200 \mathrm{~kg} \mathrm{~m}^{-3}$. The pressure drop, $\Delta P$, between the locations of the transducers is the only measured parameter for each simulation.

$$
\eta(\dot{\gamma})=k \dot{\gamma}^{n}
$$

For a constant geometry, this reduces to a relationship between 6 dimensional parameters $\left(v_{p}, \rho, k, n, R_{c}, \Delta_{P}\right)$ in 3 dimensional units $(\mathrm{kg}, \mathrm{m}, \mathrm{s})$, and hence only three independent dimensionless groups by Buckingham's $\Pi$ theorem. The power law exponent $n$ is already dimensionless, and the pressure drop can be converted into the Bagley correction factor $\alpha$ by comparing the actual pressure drop to the theoretical value predicted for an ideal capillary with no entry or exit losses, given by equation A.2[6]. Flow behaviour would be accounted for by Reynolds' number, however it was seen that the simulation values of $\alpha$ were independent of flow velocity for these flow rates and viscosities. Hence Figure A.10 shows the relationship between $n$ and $\alpha$.

$$
\alpha=\frac{\Delta P}{2 k}\left(\frac{R_{c}^{3}}{(3+1 / n) R_{p}^{2} v_{p}}\right)^{n}-\frac{L_{c}}{R_{c}}
$$




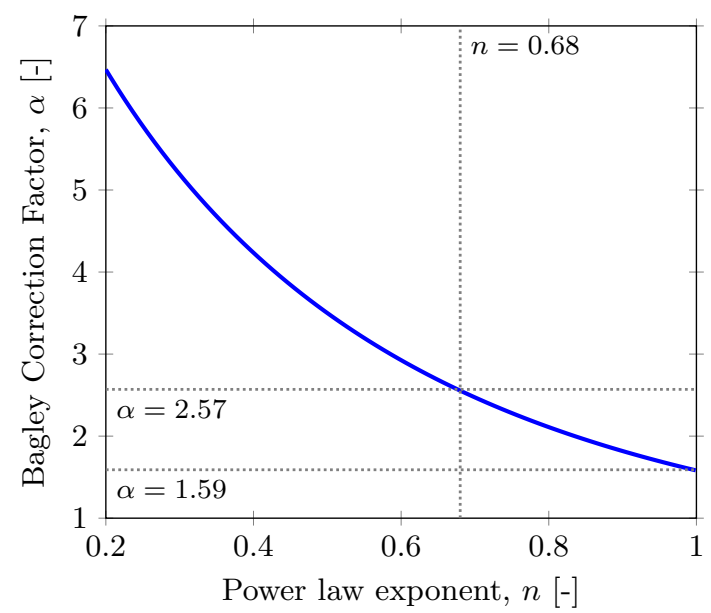

Figure A.10: Bagley correction factor

As given by the ANSYS Polyflow simulation for an ideal power law fluid in the geometry given in Figure A.9.

As the power law exponent decreases, a greater portion of the pressure drop is due to entry and exit losses. However it has been seen that molten instant coffee has both power law shear thinning behaviour and a Newtonian plateau. Therefore the actual amount of entry and exit pressure losses will depend on the flow regime, but upper and lower bounds can be obtained by looking at the fully shear thinning and fully Newtonian regimes respectively. This gives the Bagley correction factor as $\alpha=2.08 \pm 0.49$.

\section{Acknowledgements}

The authors would like to thank Dr Simon Butler for his invaluable assistance in the operation of the MPR.

Funding: This work was supported by Mondelēz International, Jacob Douwe Egberts, and the Engineering and Physical Sciences Research Council. These parties had no involvement beyond the provision of financial support.

\section{Nomenclature}

a Carreau-Yasuda transition sharpness

$\alpha$ Bagley correction factor

$\Delta P$ Pressure drop

$\dot{\gamma}$ Shear strain rate

$\dot{\gamma}_{w}$ Shear strain rate at capillary wall

$\eta_{0}$ Low shear Newtonian viscosity

$\eta_{\infty}$ High shear Newtonian viscosity

$\lambda$ Carreau-Yasuda transition time

$\tau_{w}$ Shear stress at capillary wall

$A_{\eta}$ Andrade prefractor

$k$ Powerlaw consistency index

$L_{c}$ Capillary length $n$ Shear thinning exponent

$n^{\prime}$ Rabinowitsch correction factor

$Q$ Volumetric flow rate

$R_{c}$ Capillary radius

$R_{p}$ Pistol radius

$T_{\eta}$ Andrade exponent temperature

$T_{s}$ Andrade solidification temperature

$v_{p}$ Piston velocity

\section{References}

[1] Arora, V. K., Jones, G., Kovtun, J., Gabbard, R., 1991. Evaporative process for producing coffee glass.

[2] Arora, V. K., Vitti, R. A., Rankowitz, M. M., 1992. Oil or colloidal containing gasified coffee product and process.

[3] Arora, V. K., Vitti, R. A., Rankowitz, M. M., 1995. Gasified Coffee Product and Process.

[4] Bagley, E. B., 1957. End corrections in the capillary flow of polyethylene. Journal of Applied Physics 28 (5), 624-627.

[5] Ballman, R. L., Brown, J. J., 1955. Capillary Rheometry. Instron Engineering Corporation.

[6] Bird, R. B., Stewart, W. E., Lightfoot, E. N., 1960. Transport Phenomena, 2nd Edition. John Wiley, New York.

[7] Cheremisinoff, N. P., 1996. Polymer Characterisation: Laboratory Techniques and Analysis. Noyes Publications, Westwood, New Jersey.

[8] Coventry, K. D., Mackley, M. R., 2008. Cross-slot extensional flow birefringence observations of polymer melts using a multipass rheometer. Journal of Rheology 52 (2), 401.

[9] Dealy, J. M., 1993. Rheometry for Process Control. In: Collyer, A. A. (Ed.), Techniques in Rheological Measurement. Springer, Dordrecht, Ch. 10.

[10] Fogel'son, R. L., Likhachev, E. R., aug 2001. Temperature dependence of viscosity. Technical Physics 46 (8), 1056-1059.

[11] Lee, K., Mackley, M. R., 2001. The application of the multi-pass rheometer for precise rheo-optic characterisation of polyethylene melts. Chemical Engineering Science 56 (19), 5653-5661.

[12] Lele, A., Mackley, M., Galgali, G., Ramesh, C., 2002. In situ rheo-x-ray investigation of flow-induced orientation in layered silicatesyndiotactic polypropylene nanocomposite melt. Journal of Rheology 46 (5), 1091.

[13] Mackley, M. R., 1988. Capillary Rheometry. In: Collyer, A. A., Clegg, D. W. (Eds.), Rheological Measurement, 2nd Edition. Elsevier, Ch. 1.

[14] Mackley, M. R., Marshall, R. T. J., Smeulders, J. B. A. F., nov 1995. The multipass rheometer. Journal of Rheology 39 (6), 1293.

[15] Mackley, M. R., Spitteler, P. H., 1996. Experimental observations on the pressure-dependent polymer melt rheology of linear low density polyethylene, using a multi-pass rheometer. Rheologica Acta 35, 202-209.

[16] Martin, P. J., Odic, K. N., Russell, A. B., Burns, I. W., Wilson, D. I., 2008. Rheology of commercial and model ice creams. Applied Rheology 18 (1), 2-4.

[17] Mezger, T. G., 2006. The Rheology Handbook: For users of rotational and oscillatory rheometers, 2nd Edition. Vincentz Network, Hannover.

[18] Nowjee, N. C., 2012. The Foaming Kinetics and Rheology of Water-based Potato Starch Melts. Phd, University of Cambridge.

[19] Ranganathan, M., Mackley, M. R., Spitteler, P. H. J., 1999. The application of the multipass rheometer to time-dependent capillary flow measurements of a polyethylene melt. Journal of Rheology 43 (2), 443. 
[20] Rankowitz, M. M., Epps, J., Macfeiggan, P. J. S., Scarella, R., Fave, N. D., Musto, J., Arora, V. K., Jones, G., Kovtun, J., Brandlein, L., Rudolf, V., Gabbard, R., 1990. Coffee glass and products.

[21] Roos, Y., Karel, M., 1991. Plasticizing Effect of Water on Thermal Behavior and Crystallization of Amorphous Food Models. Journal of Food Science 56 (1), 38-43.

[22] Roos, Y. H., 1998. Phase transitions and structure of solid food matrices. Current Opinion in Colloid \& Interface Science 3 (6), 651-656.

[23] Sivetz, M., Foote, H. E., 1963. Coffee processing technology, volume 1. Vol. 1. Avi Pub. Co., Westport, Conn.,.

[24] Steffe, J. F., 1992. Rheological Methods in Food Process Engineering, 2nd Edition. Freeman Press, East Lansing.

[25] Tanner, R., Walters, K., 1998. Rheology: An Historical Perspective. Elsevier, Amsterdam.

[26] Taylor, J. E., Van Damme, I., Johns, M. L., Routh, A. F., Wilson, D. I., 2009. Shear rheology of molten crumb chocolate. Journal of Food Science 74 (2), 55-61.

[27] Technavio, 2015. A look and instant coffee pricing and the global market landscape.

[28] Tuladhar, T. R., Mackley, M. R., 2004. Experimental observations and modelling relating to foaming and bubble growth from pentane loaded polystyrene melts. Chemical Engineering Science 59 (24), 5997-6014.

[29] Washington Post, 2014. Almost half of the world actually prefer instant coffee.

[30] White, D. E., Moggridge, G. D., Ian Wilson, D., 2008. Solidliquid transitions in the rheology of a structured yeast extract paste, Marmite TM. Journal of Food Engineering 88 (3), 353363.

[31] Zeller, B. L., Ceriali, S., Gundle, A., 2012. Foaming soluble coffee powder containing pressurized gas. 